\title{
Characterization of a de novo SSMC 17 detected in a girl with developmental delay and dysmorphic features
}

\author{
Lana Stavber', Sara Bertok², Jernej Kovač ${ }^{1}$, Marija Volk³ ${ }^{3}$ Luca Lovrečić $^{3}$, Tadej Battelino ${ }^{2,4}$ and Tinka Hovnik ${ }^{*}$
}

\begin{abstract}
Background: The majority of small supernumerary marker chromosome cases arise de novo and their frequency in newborns is $0.04 \%$. We report on a girl with developmental delay and dysmorphic features with a non-mosaic de novo SSMC that originated from the pericentric region of q arm in chromosome 17.

Case presentation: The girl presented with developmental delay, speech delay, myopia, mild muscle hypotonia, hypoplasia of orbicular muscle, poor concentration, and hyperactivity. Main dysmorphic features included: round face, microstomia, small chin, down-slanting palpebral fissures and small lobules of both ears. At present, her developmental abilities are still delayed for her chronological age but she is making evident progress with speech.

A postnatal array comparative genomic hybridization showed a $2.31 \mathrm{Mb}$ genomic gain indicating microduplication derived from pericentric regions q11.1 and q11.2 of chromosome 17. Additional conventional cytogenetic analysis from peripheral blood characterized the karyotype as 47,XX,+mar in a non-mosaic form. The location of microduplication was confirmed with fluorescence in situ hybridization.
\end{abstract}

Conclusion: The proband's microduplication encompassed approximately 40 annotated genes, several of which have been associated with phenotypic characteristics of the proband. This is the first report of SSMC 17 including this particular chromosomal region in non-mosaic form.

Keywords: Small supernumerary marker chromosome, Developmental delay, Speech delay, Dysmorphic features, fluorescence in situ hybridization

\section{Background}

Small supernumerary marker chromosomes (sSMC) are a morphologically heterogeneous group of structurally abnormal chromosomes that cannot be identified unambiguously by conventional cytogenetics. The definite origin of sSMC can only be diagnosed using molecular cytogenetic techniques [1]. sSMC frequency in newborns is $0.04 \%$ [2] but their clinical variability may range from normal to severely abnormal phenotype. The effects of sSMC may be attributed to their size, presence of euchromatic material and degree of mosaicism [3]. Uniparental disomy of sSMC's sister chromosomes can also be identified [4]. The majority of sSMC cases arise de novo whilst $30 \%$ segregate within a family [3].

\footnotetext{
* Correspondence: tinka.hovnik@kclj.si

'University Children's Hospital, University Medical Centre Ljubljana, Unit for

Special Laboratory Diagnostic, Vrazov trg 1, SI-1525 Ljubljana, Slovenia

Full list of author information is available at the end of the article
}

A de novo sSMC derived from a pericentric region of the $\mathrm{q}$ arm in chromosome 17 in a centric minute shape is described. It was identified postnatally and confirmed with fluorescence in situ hybridization (FISH). To our knowledge, this is the first report of this particular sSMC 17 in non-mosaic form.

\section{Case presentation}

The 4 years old proband was born after 36 weeks of gestation to non-consanguineous healthy parents. The proband's birth weight was $2600 \mathrm{~g}$ (10th-25th percentile), birth length $47 \mathrm{~cm}\left(25^{\text {th }}-50\right.$ th percentile), occipital-frontal circumference $32 \mathrm{~cm}$ (25th-50th percentile).

At the age of two months gastroesophageal reflux was diagnosed. She walked unaided at 18 months and started uttering her first words at the age of 3 years, when her pediatrician referred her for further development assessment. Her craniofacial features included round face, 
microstomia, small chin and down-slanting palpebral fissures. Lobules of both ears were small. Ultrasound examination of the heart and lung was unremarkable. Metabolic, endocrine and biochemical screening results were normal. Ophthalmologic examination showed myopia and anisometropia. Neurologic examination revealed mild general hypotonia with hypoplasia of orbicular oris muscle and levatorangulioris muscle. Her overall speech skills were delayed for at least 1.5 years. Her comprehension was limited to simple commands. Speech therapist was involved for further speech evaluation. She had a problem with concentration and visual-motoric coordination. Psychological examination revealed poor social interactions and severe hyperactivity. Other abnormal findings were supernumerary nipple on the left side, proximal placement of the thumbs and polycystic ovary. Dermatogliphes were normal; she had a small hypopigmented skin lesion on right thigh.

From neonatal period to the present age she didn't have significant health problems except recurrent otitis media. At first she attended kindergarten for children with developmental problems, but recently joined a regular kindergarten with additional speech and occupational therapy.

Once a year she is re-evaluated by the clinical geneticist. Her developmental abilities are constantly improving but are still delayed for her biological age. At the age of 4 she uses limited number of words in few-word sentences and understands simple commands. Her speech is sometimes still unclear and problems with hyperactivity persist. At the age of 4.2 years her developmental quotient (DQ) was calculated to be 0.68 , representing significant development delay.

\section{Molecular and cytogenetic analysis}

All cytogenetics and molecular-genetics analyses were performed with written and signed informed consent. Cytogenetic studies were carried out on peripheral blood lymphocytes from the patient and her parents. Cytogenetic analyses on metaphase-spread preparations were performed with standard procedures using GTG banding at a minimum 500 band resolution level according to the International System for Human Cytogenetic Nomenclature (ISCN 2013). Further molecularcytogenetics analyses were undertaken using the FISH probe for chromosomal region 17q11.2 RP11-192H23 (BlueGnome, Illumina, San Diego, CA) on metaphase and interphase chromosome spreads. Chromosomes were counterstained with 4;6-diamidino-2-phenylindole (DAPI) and images were captured using the CytoVysion Imaging System (Leica Biosystems. Wetzlar, DE).

Array comparative genomic hybridization (aCGH) analysis was performed on genomic DNA from a proband's peripheral blood sample using a commercial oligonucleotide array (Agilent $60 \mathrm{~K}$ ISCA Oligo, Agilent Technologies, Santa Clara, CA) and sex-matched human reference DNA sample (Agilent Technologies, Santa Clara, CA). Data were analysed with the Cytogenomics 3.0 Software (Agilent Technologies, Santa Clara, CA). Peripheral blood lymphocytes from both parents were analysed by conventional karyotyping and additionally using specific 17q11.2 FISH probe RP11-192H23 to exclude the parental presence of sSMC or a balanced chromosomal rearrangement.

\section{Results}

A postnatal aCGH showed a microduplication of $2.31 \pm$ $0.06 \mathrm{Mb}$ encompassing pericentric regions q11.1 and q11.2 derived from chromosome 17 (arr[hg19] 17q11.1q11.2(25,403,446-27,716,930)x3) (Fig. 1). Based on cytogenetic analysis of metaphases from peripheral blood we interpreted the karyotype as 47,XX,+der(17)(:p11.1- > q11.2:) in non-mosaic form. The location of microduplication was confirmed with molecular analysis using FISH probe RP11-192H23 (Fig. 2). Based on its shape, size and centromere we concluded that marker chromosome was a centric minute sSMC 17. Parental analysis demonstrated normal karyotypes and regular chromosomes $17 \mathrm{FISH}$ patterns, consistent with a de novo origin of sSMC.

\section{Discussion}

We found a de novo sSMC derived from chromosome region 17q11.1q11.2 using aCGH and FISH in a girl with developmental delay, speech delay, dysmorphic features and hyperactivity. According to the SSMC 17 database at Jena University Hospital, the proximal partial trisomy of q arm of chromosome 17 in general leads to developmental delay, dysmorphism, growth retardation, hypotonia, and visual impairment [5]. All these characteristics were observed in our proband. The duplicated region of cytoband 17q11.2 lies outside the potentially non dosage-sensitive pericentric region as confirmed by molecular mapping listed in SSMC database.

The formation of this de novo sSMC was probably due to a partial trisomy rescue and may be the result of viable postzygotic nondisjunction or anaphase lag event occurring during early embryogenesis $[3,6]$. Advanced maternal age increases the incidence of meiotic non-disjunction events [7] and in our case the mother was 33 years old at the time of conception. There are numerous proposed models for centric minute sSMC formation but so far no pathways or enzymes involved in the processes of trisomy rescue mechanisms were discovered [3]. Although the majority of this life saving outcomes result in mosaic sSMC formation, the discrepancy in the level of mosaicism in different tissues of the same individual is well established. Trisomy rescue is also one of proposed mechanism for uniparental disomy (UPD). It has been estimated that de novo sSMC contribute to $4 \%$ of reported UPD cases [8]. However, UPD 17 is not regarded to be associated with 


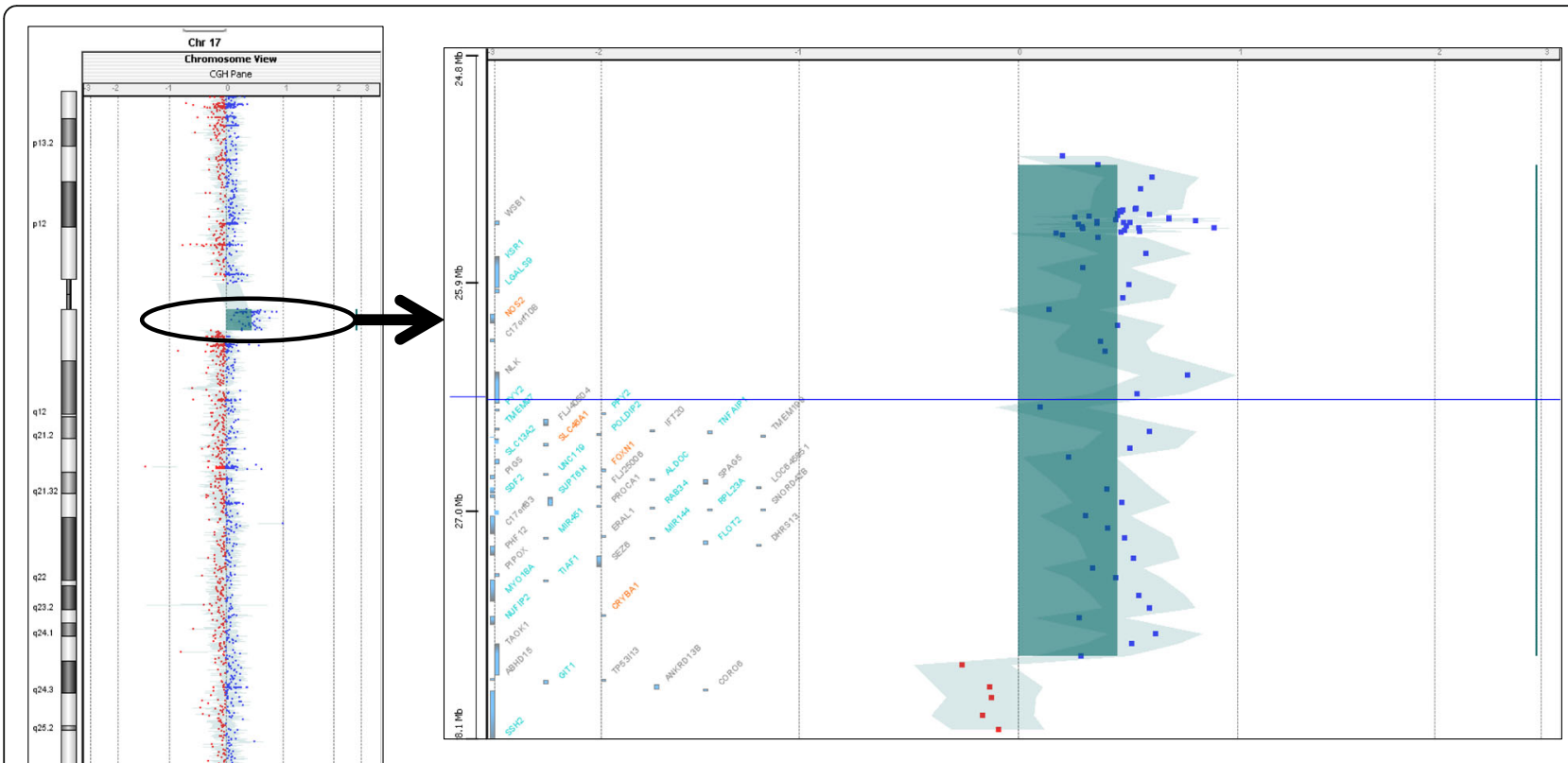

Fig. 1 The result of aCGH indicating microduplication of chromosome 17 (arr[hg19]17q11.1q11.2(25,403,446-27,716,930)x3). Whole chromosome 17 and a close-up of duplicated region with annotated genes is visualized

any imprinting disorder. To date more than 3.300 cases have been reported in UPD database, yet there are no reports for coincidence of sSMC and mat/pat UPD 17 with clinical finding. Therefore, we interpreted this minute centric de novo sSMC 17 as resulting from a combination of meiosis non-disjunction event and incomplete trisomy rescue event in which the majority of additional chromosome 17 was lost. The remaining minute sSMC 17 harbours genomic gain which is entirely causative for the clinical phenotype.
The proband's microduplication encompassed approximately 40 annotated OMIM genes, several of which were reported as potentially associated with proband's phenotypic characteristics. Among clinically relevant genes within the duplicated region were NOS2, TRAF4, SEZ6, SARM1 and LGALS9. Gene NOS2 (OMIM $163730)$ encodes nitric oxidase synthase $2 \mathrm{~A}$ that produces nitric oxide (NO), an important second messenger molecule involved in the regulation of cardiovascular, immune and neural system. NO has an array of functions in

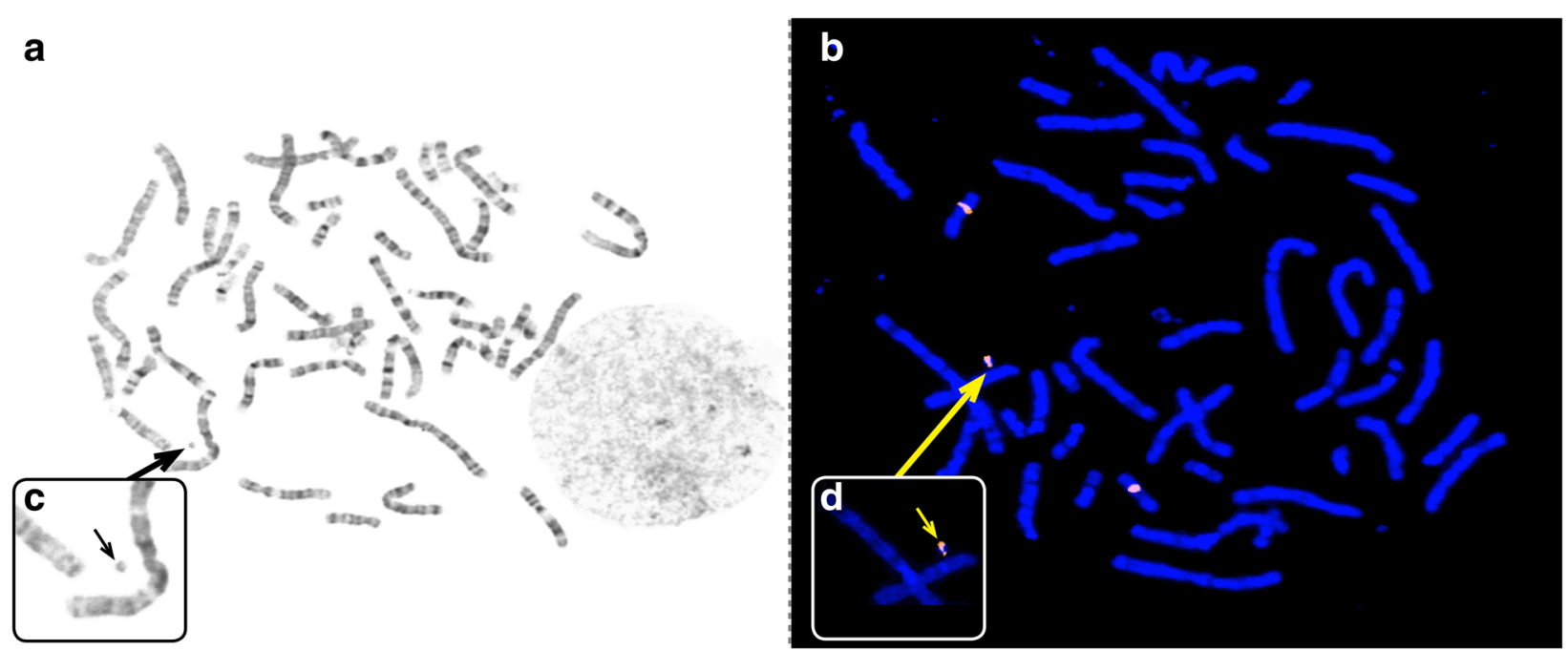

Fig. 2 Results of high resolution G-banding (a) and FISH (b) with identified SSMC marked by arrows. The close-up of sSMC for corresponding metaphase is presented in both smaller frames (c, d). The orange FISH signals are the result of metaphase hybridization with the probe RP11-192H23 (BlueGnome, Illumina) 
the central nervous system. In an association with cognitive function it has an important role in the induction and maintenance of synaptic plasticity [9]. Furthermore, the excess production of NO causes "nitrosative stress" and consequent neurotoxicity. NO also acts at the level of transcription and translation and regulates cell survival and proliferation in diverse cell types, including neuron cells [10]. So, it affects neurogenesis, where the continuous formation and pruning of neuron synapses are the main processes. Second gene TRAF4 (OMIM 602464) encodes TNF receptor-associated factor 4 , which is required during embryogenesis for the formation of the trachea, the axial skeleton and the closure of neural tube. In addition, TRAF4 plays a role in proper myelination therefore its role in ADHD, intellectual disability and movement disorder has been previously suggested [11]. Other genes within the duplicated region were also of interest for possible genotypephenotype correlations. Gene SEZ6 (OMIM 616666) is predicted to be involved in neuronal maturation and plasticity, based on animal studies [12]. Mary et al. reported that SEZ6 expression correlates with the most active periods of cortical neurogenesis and neuronal maturation [13]. Gene SARM1 (OMIM 607732) activity is required after axon injury to induce axon degeneration in mice [14], while LGALS9 gene (OMIM 601879) encodes different lectins. Specific interactions between carbohydrate moieties and their putative binding proteins (i.e. lectins) play a critical role in various developmental, physiologic, and pathologic processes. Based on the description and the mechanism of genes included in this SSMC, we suggest that duplication of NOS2 and TRAF4 genes play a crucial role in our patient. Altered NO production in brain may affect neurotoxicity and eventually influence neurogenesis. It results in an inappropriate development and working of the whole central nervous system, which could be a reason for the proband's developmental delay. It has been shown that TRAF4 is attractive gene for movement disorders [11], so we can suggest that duplication of TRAF4 is probably involved in our patient's hyperactivity. This observation remains speculative and needs to be confirmed in other similar cases or functional studies. Currently, there are no published reports for SEZ6, SARM1 and LGALS9 involvement into development of developmental delay or dysmorphism and their role in the etiology of presented case is unclear. The comprehensive study of all annotated genes in the duplicated region was performed but no candidate genes for supernumerary nipple or polycystic ovary was identified. However, the function of many genes in the duplicated region is currently not known.

To date, not a single reported case with the SSMC 17 encompassing this particular coordinates of pericentric region of q11.1 and q11.2 was identical to ours. In Table 1 we present overlapping cases from the literature. The presence of sSMC 17 encompassing only the pericentric regions appears to be associated predominantly with developmental delay and in some cases with

Table 1 Clinical cases of sSMC 17

\begin{tabular}{|c|c|}
\hline Author & Final result of SSMC 17 \\
\hline Chen et al. & $\begin{array}{l}17 q 11.1 q 11.2(25,372,965-27,725,134) \\
\text { in mosaic form; prenatal diagnosis }\end{array}$ \\
\hline Cornelius et al. & 17p11.2q11.2 $(21,200,000-27,500,000)$ in mosaic form \\
\hline Vetro et al. & $\begin{array}{l}\text { sSMC(17) involving three duplications separated by two } \\
\text { single copy regions with a size of about } 2.1 \mathrm{Mb} \text { and } 615 \\
\text { kb (NCBI 36): 17p11.2 (16,892,427-19,888,467) (2,9 Mb), } \\
17 \mathrm{q} 11.1(22,427,573-23,163,556)(319 \mathrm{~kb}) \text { and } 17 \mathrm{q} 11.2 \\
(23,848,894-25,676,268)(1,8 \mathrm{Mb}) \text { in mosaic form }\end{array}$ \\
\hline
\end{tabular}

Clinical symptoms
language problems at neonatal follows-up

22-year-old male with Gilles de la Tourette syndrome, attention deficit [11] hyperactivity disorder (ADHD), intellectual disability and seizures

severe global developmental delay, speech delay, hypotonia, microcephaly and mild dysmorphic features

Kozma et al. $r(17)(:: p 11.1 \rightarrow q 21::)$ in mosaic form; no coordinates given

38-year-old male with developmental delay, profound mental retardation, kyphoscoliosis, bilateral cataracts, severe calcaneovalgus deformity of the feet, dysmorphic facies, mitral valve prolapse with regurgitation and severe respiratory insufficiency. He never developed any speech.

Capovia P. et al. $\min (17)(: p 11.1 / q 11.2:)$ in size of $10 \mathrm{Mb}$; in mosaic form

2-year-old male with minor facial dysmorphic features, developmental delay (especially speech delay), short stature and Potocki-Lupski syndrome

Jason Anderson $\min (17)(23,086,100-32,754,790 \mathrm{MB})$

Manolakos et al. $\min (17)$ of three duplications: $(\mathrm{p} 11.2 \rightarrow \mathrm{p} 11.2 ; 16.9-19.89)$ $(p 11.1 \rightarrow q 11.1 ; 22.42-23.16)(q 11.2 \rightarrow q 11.2 ; 23.84-25.67)$ in non-mosaic form developmental delay in newborn

significantly delayed developmental milestones (sitting unsupported at 12 months, walking unaided at 22 months, started uttering first words at 2.5 years), mild dysmorphic facial and body features (microcephaly, narrow palpebral fissures, small eyes, high-arched palate, low set ears, short hands and fingers and clinodactyly of the 5th finger), severe hypotonia, microcephaly, IQ $<46$ (at 17 months)

abnormal 
intellectual disability, meanwhile patients with sSMC 17 of larger parts of either the short or the long arm of chromosome 17 have additional dysmorphic features [11]. The duplicated genomic region of the present case is the most comparable to that of Chen et al.[15], although our proband's heart examination did not reveal any abnormalities. Both cases manifested with developmental delay and speech delay, despite the fact that sSMC 17 in the Chen et al. case was in a mosaic form. Cornelius et al. described another similar case [11] and suggested that the duplication of NOS2 plays a role in the ADHD symptoms of their patient. Cornelius et al. also suggested that the duplication of TRAF4 plays a role in ADHD, intellectual disability and movement disorder. Although the present proband does not fulfill all the criteria of ADHD, she has a severe form of hyperactivity.

\section{Conclusion}

We report a de novo sSMC derived from 17q11.1q11.2 in association with developmental delay, speech delay and mild dysmorphism. This unique clinical case was compared to published overlapping cases. We conclude that the identification of the origin, the size and the composition of sSMC using aCGH and FISH are essential for precise genotype-phenotype correlation. Furthermore, thorough molecular-cytogenetic characterization of sSMC is needed for the prediction of clinical prognosis and genetic counseling.

\section{Abbreviations}

aCGH: Array comparative genomic hybridization; SSMC: Small supernumerary marker chromosome; FISH: Fluorescent in situ hybridization; GTG: GiemsaTripsin-Gbanding

\section{Acknowledgements}

We thank Ms Maja Ficko and Ms Vanda Matuc for their expert technical assistance. We also thank the family for their collaboration in this study.

\section{Funding}

The study was supported in part by the Slovene National Research Agency grants J3-2413 and P3-0343.

\section{Availability of data and materials}

The datasets analyzed during the current study are available from the corresponding author on reasonable request. This case was submitted to the sSMC database at Jena University Hospital, No. 17-W-p11.1/4-2 (http://ssmc-tl.com/ chromosome-17.html).

\section{Authors' contributions}

$\mathrm{TH}, \mathrm{JK}$ and SB performed the molecular cytogenetic studies in the present case and collected the data relative to this case report. LL and MV did the array-CGH analysis, interpretations and reviewed the manuscript. SL in $\mathrm{TH}$ drafted the paper and TB with all authors contributed to the finalizing of the manuscript. All authors read and approved the final manuscript.

\section{Competing interests}

The authors declare that they have no competing interests.

\section{Consent for publication}

Written informed consent was obtained from the parents of the patient for the publication of this case report.

\section{Ethics approval and consent to participate}

Written informed consent was obtained from all participants prior to the genetic study. The protocol was approved by the Slovene Medical Ethics Committee. The study followed the principles of the Declaration of Helsinki.

\section{Publisher's Note}

Springer Nature remains neutral with regard to jurisdictional claims in published maps and institutional affiliations.

\section{Author details}

${ }^{1}$ University Children's Hospital, University Medical Centre Ljubljana, Unit for Special Laboratory Diagnostic, Vrazov trg 1, SI-1525 Ljubljana, Slovenia. ${ }^{2}$ Department of Pediatric Endocrinology, Diabetes and Metabolic Diseases, University Children's Hospital, UMC, Ljubljana, Slovenia. ${ }^{3}$ Clinical Institute of Medical Genetic, UMC, Ljubljana, Slovenia. ${ }^{4}$ Faculty of Medicine, University of Ljubljana, Ljubljana, Slovenia.

Received: 13 January 2017 Accepted: 17 March 2017

Published online: 23 March 2017

\section{References}

1. Liehr T, Claussen U, Starke H. Small supernumerary marker chromosomes (sSMC) in humans. Cytogenet Genome Res. 2004;107:55-67.

2. Liehr T, Weise A. Frequency of small supernumerary marker chromosomes in prenatal, newborn, developmentally retarded and infertility diagnostics. Int J of Mol Med. 2007;19:719-31.

3. Liehr T. Small Supernumerary Marker Chromosomes: A Guide for Human Geneticist and Clinicians. Berlin Heidelberg: Springer Verlag; 2012.

4. Röthlisberger B, Zerova T, Kotzot D, Buzhievskaya TI, Balmer D, Schinzel A. Supernumerary marker chromosome (1) of paternal origin and maternal uniparental disomy 1 in a developmentally delayed child. J Med Genet. 2001;38:885-8.

5. Liehr T. Small supernumerary marker chromosomes. http://ssmc-tl.com/ chromosome-17.html. Accessed Feb 2017.

6. Bartels I, Schlueter G, Liehr T, von Eggeling F, Starke H, Glaubitz R, Burfeind $P$. Supernumerary small marker chromosome (SMC) and uniparental disomy 22 in a child with confined placental mosaicism of trisomy 22: trisomy rescue due to marker chromosome formation. Cytogenet Genome Res. 2003;101(2):103-5

7. Shinzel A. Catalogue of unbalanced chromosome aberrations in men. Berlin: Walter de Gruyter; 2001.

8. Liehr T. Cytogenetic contribution to uniparental disomy (UPD). Mol Cytogenet. 2010;3:8.

9. Calabrese V, Mancuso C, Calvani M, Rizzarelli E, Butterfield DA, Stella AM. Nitric oxide in the central nervous system: neuroprotection versus neurotoxicity. Nat Rev Neurosci. 2007:8:766-75. doi:10.1038/nrn2214.

10. Beltran-Povea A, Caballano-Infantes E, Salquero-Aranda C, Martín F, Soria B, Bedoya FJ, Tejedo JR, Cahuana GM. Role of nitric oxide in the maintenance of pluripotency and regulation of the hypoxia response in stem cells. World J Stem Cells. 2015;7(3):605-17.

11. Cornelius N, Bertelsen B, Melchior L, Nazaryan L, Debes NM, Groth C, Skov L, Tümer Z. A mosaic small supernumerary marker chromosome 17 in a patient with Tourette syndrome, ADHD and intellectual disability: a case story and review of the literature. Psychiatry Res. 2015;228:179-81.

12. Miyazaki T, Hashimoto K, Uda A, Sakagami H, Nakamura Y, Saito S, Nishi M, Kume H, Tohgo A, Kaneko I, Kondo H, Fukunaga K, Kano M, Watanabe M, Takeshima $\mathrm{H}$. Disturbance of cerebellar synaptic maturation in mutant mice lacking BSRPs, a novel brain-specific receptor-like protein family. FEBS Lett. 2006;580:4057-64

13. Kim MH, Gunnersen JM, Tan SS. Localized expression of the seizure-related gene SEZ-6 in developing and adult forebrains. Mech Dev. 2002;118:171-4. doi:10.1016/S0925-4773(02)00238-1.

14. Gerdts J, Brace EJ, Sasaki Y, DiAntonio A, Milbrandt J. SARM1 activation triggers axon degeneration locally via NAD+ destruction. Science. 2015;348:453-7.

15. Chen CP, Chiang S, Wang KL, Cho FN, Chen M, Chern SR, Wu PS, Chen YN, Chen SW, Chang SP, Chen WL, Wang W. Prenatal diagnosis of mosaic small supernumerary marker chromosome 17 associated with ventricular septal defect, developmental delay, and speech delay. Taiwan J Obstet Gynecol. 2016;55:419-22. 
16. Vetro A, Manolakos E, Petersen M, Thomaidis L, Liehr T, Croci G, Franchi F, Marinelli M, Meneghelli E, Dal Bello B, Cesari S, lasci A, Arrigo G, Zuffardi O. Unexpected results in the constitution of small supernumerary marker chromosomes. Eur J Med Genet. 2012;55:185-90. doi:10.1016/j.jmg. 2012.01.010.

17. Kozma C, Blancato J, Meck J, Jiang Y. Characterization of a Supernumerary Marker Derived From Chromosome 17 by Microdissection in an Adult With MR/MCA. Am J Med Genet. 1998;77:19-22.

18. Capovia P, Godava M, Hyjanek J, Hajduch M, Santavy J. Potocki-Lupski syndrome and Turner syndrome in two patients with mosaicism for a marker chromosomes. Chromosome Res. 2011;19 Suppl 1:p S37. Abstractnr. 1. P02 - information from poster.

19. Manolakos E, Thomaidis L, Lagou M, Neroutsou R, Kefalas K, Louizou E, Rapti M, Kontodiou M, Triga N, Tsoplou P, LiehrT, Petersen MB, Metaxotou A. Supernumerary minute chromosome 17 in a boy with severe developmental delay: molecular breakpoint in the unstable proximal 17p region. Europ J Hum Genet. 2010;18(Suppl1):113 (Abstractnr. P03.047).

20. Neill NJ, Torchia BS, Bejjani BA, Shaffer LG, Ballif BC. Comparative analysis of copy number detection by whole-genome BAC and oligonucleotide array CGH. Mol Cytogenet. 2010;3:11

Submit your next manuscript to BioMed Central and we will help you at every step:

- We accept pre-submission inquiries

- Our selector tool helps you to find the most relevant journal

- We provide round the clock customer support

- Convenient online submission

- Thorough peer review

- Inclusion in PubMed and all major indexing services

- Maximum visibility for your research

Submit your manuscript at www.biomedcentral.com/submit
Biomed Central 